\title{
Análisis de los transitorios de ruido electroquímico para aceros inoxidables 316 Y - DUPLEX 2205 en $\mathrm{NaCl}$ Y FeCI(')
}

\author{
F. Almeraya-Calderón"***, F. Estupiñán ${ }^{* *}$, P. Zambrano R*, A. Martínez-Villafañe ${ }^{* *}$, A. Borunda T**, \\ R. Colás $\mathrm{O}^{*}$ y C. Gaona-Tiburcio*
}

\begin{abstract}
Resumen En este trabajo se muestran los resultados obtenidos de las mediciones de ruido electroquímico para diferentes materiales que exhiben corrosión por picaduras. Los transitorios presentados en las series de tiempo en corriente y en potencial, se correlacionan con el análisis superficial de microscopía electrónica de barrido (MEB). Las mediciones electroquímicas fueron realizadas a diferentes tiempos de exposición, para obtener una correlación. Los materiales usados fueron los aceros inoxidables 316 y dúplex 2205 , inmersos en cloruro férrico $\left(\mathrm{FeCl}_{3}\right)$ y cloruro de sodio $(\mathrm{NaCl})$ como electrolitos. Los análisis por MEB, muestran que los transitorios observados en las series de tiempo, corresponden realmente con la actividad de la nucleación de picaduras desarrollada sobre la superficie de los electrodos.
\end{abstract}

Palabras clave Corrosión por picaduras; Ruido electroquímico; Técnicas electroquímicas; Acero inoxidable austenítico 316; Acero inoxidable Dúplex 2205.

\section{Electrochemical noise transient analysis for 316 and Duplex 2205 stainless steels in $\mathrm{NaCl}$ and $\mathrm{FeCl}$}

\begin{abstract}
This work shows the results obtained from electrochemical noise measurements for different materials exhibiting pitting corrosion. The transients presented in the potential and current time, correlates with the scanning electron microscopy (SEM) surface analysis. Electrochemical measurements were made at different exposure times to obtain the correlation. The materials used were stainless steel austenitic 316 and duplex 2205, immersed in ferric chloride $\left(\mathrm{FeCl}_{3}\right)$ and sodium chloride $(\mathrm{NaCl})$ electrolytes. SEM analysis shows that the transients observed in the time series, really correspond to the activity of pit nucleation developed over the surface of the electrodes.
\end{abstract}

Keywords

\section{INTRODUCCIÓN}

La película de óxido formada en la superficie de materiales como los aceros inoxidables, así como de aleaciones base níquel, aluminio, titanio, cobre y otros metales, es la responsable de su alta resistencia a la corrosión. Sin embargo, la resistencia de la película pasiva está determinada por las condiciones ambientales a que está expuesto el material, así como por la composición de la aleación ${ }^{[1]}$. $\mathrm{Al}$ incrementar la concentración de los iones agresivos, especialmente iones cloruro $\mathrm{Cl}^{-}$, también aumenta el ataque por picaduras ${ }^{[2]}$, y causan que las películas pasivas sean susceptibles al rompimiento localizado, resultando en una disolución acelerada del metal ${ }^{[3]}$, principalmente en los sitios donde existen irregularidades en la superficie del material, produciendo la corrosión localizada, principalmente corrosión por picaduras en los aceros inoxidables ${ }^{[4}$ y 5$]$.

Como parte de las técnicas electroquímicas, se encuentra el Ruido Electroquímico (EN), que es una técnica no destructiva ${ }^{[6]}$, y que refleja la suma individual de los eventos aleatorios de las fluctuaciones

\footnotetext{
(•) Trabajo recibido el día 1 de diciembre de 2011 y aceptado en su forma final el día 20 de enero de 2012.

* Universidad Autónoma de Nuevo León (UANL). Facultad de Ingeniería Mecánica y Eléctrica (FIME) - Centro de Investigación e Innovación en Ingeniería Aeronáutica (CIIIA). Av. Universidad s/n. Ciudad Universitaria. San Nicolás de los Garza, Nuevo León, C.P.66451, México. E-mail: citlalli.gaona@gmail.com.

** Centro de Investigación en Materiales Avanzados S.C (CIMAV), Departamento de Integridad y Diseño de Materiales Compuestos Grupo Corrosión. Miguel de Cervantes 120, Complejo Industrial Chihuahua, 31109 Chihuahua, México.
} 
del potencial y/o de la corriente de un material sujeto a condiciones de corrosión ${ }^{[7-11]}$. El valor de la raíz media cuadrática (RMS) de la amplitud de estos eventos o desviación estándar se ha visto que provee la huella dactilar de la cantidad de metal disuelto, dependiendo de la combinación metal-medio ambiente. Las fluctuaciones parecen estar ligadas a variaciones en la velocidad de las reacciones anódica y catódica, como consecuencia de los procesos estocásticos (rompimiento y repasivación de la película pasiva) y determinísticos (formación y propagación de picadura) $)^{[12-14]}$.

Con la técnica de EN no se altera el estado del sistema en estudio, ya que no se aplica ninguna perturbación externa para realizar las mediciones ${ }^{[15]}$. El concepto de ruido electroquímico presenta un comportamiento inversamente proporcional al intervalo de frecuencia de las oscilaciones, a menor frecuencia mayor amplitud, pudiendo los procesos de corrosión ser investigados mediante la observación del Ruido Electroquímico ${ }^{[8-16]}$. En el caso de la corrosión por picaduras, el cual es un caso especial de la corrosión localizada, existen varios métodos para el análisis de los datos de ruido, entre los más populares están los métodos estadísticos como el índice de picado y la densidad de potencia espectral (o análisis espectral). Las fluctuaciones usualmente son medidas por un arreglo de tres electro$\operatorname{dos}^{[17}$ y 18$]$.

En este trabajo se analizan los transitorios observados en las series de tiempo de potencial y corriente correspondientes a las mediciones de ruido electroquímico, y se evaluó su correlación con las fotomicrografías obtenidas por MEB de los aceros inoxidables 316 y dúplex 2205, en solución acuosa de $3,5 \% \mathrm{NaCl}_{\text {y }} 6 \mathrm{FeCl}_{3}$.

\section{METODOLOGIA EXPERIMENTAL}

En este trabajo se utilizó el acero inoxidable austenítico 316 y el acero dúplex austenítico - ferrítico
2205. El análisis químico (cualitativo y cuantitativo) se realizó mediante Espectrometría de Emisión por Plasma. La composición química de estos aceros se muestra en la tabla I.

Para la preparación de los electrodos, estos fueron cortados de una barra de acero inoxidable austenítico AISI 316 y dúplex 2205 de $1,13 \mathrm{~cm}$ de diámetro, uniéndose a un alambre de cobre sin aislante en los extremos, para realizar el contacto eléctrico al momento de realizar los ensayos electroquímicos, y se encapsularon en resina epóxica. El área de exposición de las muestras de acero en las soluciones de prueba, fue de $1 \mathrm{~cm}^{2}$. Una vez montados, los electrodos fueron desbastados mediante una lija de grado 600, enjuagados con agua destilada y secados con alcohol y aire caliente a presión. Los electrodos fueron guardados en un desecador por un tiempo de $24 \mathrm{~h}$. antes de realizar las pruebas electroquímicas.

Como se mencionó previamente, los electrolitos empleados fueron cloruro férrico $\left(\mathrm{FeCl}_{3}\right)$ al $6 \%$ y cloruro de sodio $(\mathrm{NaCl})$ al 3,5\%, siendo preparados con reactivos químicos y agua destilada. Las pruebas electroquímicas se realizaron por inmersión en un vaso de precipitado y a $25^{\circ} \mathrm{C}$. Se emplearon dos electrodos de trabajo nominalmente idénticos ${ }^{[19}$ y 20$]$, uno como electrodo de trabajo y otro como electrodo auxiliar. El electrodo de referencia empleado fue el de calomel saturado.

Para las mediciones de ruido electroquímico se empleó un potenciostato / galvanostato /ZRA marca "Solartron 1285", y fueron llevadas a cabo una vez que el potencial a circuito abierto se estabilizó ${ }^{[21]}$. Para cada medición se muestrearon de 500 a $1.000,2.500$ y 4.096 puntos, con una velocidad de 1 punto/s. Para cada uno de los experimentos se emplearon electrodos nuevos. Una vez finalizada la prueba electroquímica, las muestras se secaron con alcohol y aire caliente a presión, y se guardaron en un desecador para posteriormente realizar el análisis por MEB. En el análisis por MEB se realizó un barrido por toda la superficie de la

Tabla I. Composición química de los materiales. (\% en peso)

Table I. Chemical composition of materials (\% weigth)

\begin{tabular}{lcccccccc}
\hline \multirow{2}{*}{ Material } & \multicolumn{10}{c}{ Elementos } \\
\cline { 2 - 9 } & $\mathbf{C}$ & $\mathbf{C r}$ & $\mathbf{S i}$ & Mn & Mo & Ni & N & Fe \\
\hline SS 316 & 0,08 & 16,65 & 0,69 & 1,47 & 2,094 & 10,45 & \multirow{2}{*}{$* * *$} & \multirow{2}{*}{ Balance } \\
Dúplex 2205 & 0,028 & 23,012 & 0,44 & 1,6 & 2,973 & 5,876 & 0,165 & Balance \\
\hline
\end{tabular}


muestra para identificar nucleaciones o picaduras presentes.

\section{RESULTADOS Y DISCUSIÓN}

En la figura 1 se muestran las series de tiempo en potencial y corriente, para las mediciones de ruido electroquímico con 500, 1.000, 2.500 y 4.096 datos, correspondientes a las mediciones para el acero inoxidable dúplex 2205 en presencia de $\mathrm{FeCl}_{3}$ al $6 \%$ en peso. En la serie de tiempo en potencial se observa una tendencia al ennoblecimiento, y por lo tanto la pasivación por parte del material bajo estudio, iniciando con potenciales activos y observando una tendencia hacia potenciales más nobles. También presenta transitorios de baja amplitud y pronta recuperación, siendo estos de forma semiexponencial.

Los transitorios súbitos de corriente han sido observados, y asociados a la nucleación de sitios localizados de picadura, picaduras metaestables o en algunos casos a corrosión por hendidura o crevice. De acuerdo con lo observado, y viendo que el potencial continuó con su tendencia hacia valores más nobles, se puede suponer que estas picaduras nuclearon o son metaestables, pero no necesariamente significa que se propagó el ataque ${ }^{[9}$ y 22$]$.

Sin importar el número de datos tomados, la tendencia de las fluctuaciones del potencial o de la corriente en el acero inoxidable dúplex 2205 es el mismo (Figs. 1 y 2). En ambas series se observan transitorios anódicos. Se puede identificar claramente que los transitorios en corriente tienen una alta frecuencia y tiempos de duración muy cortos

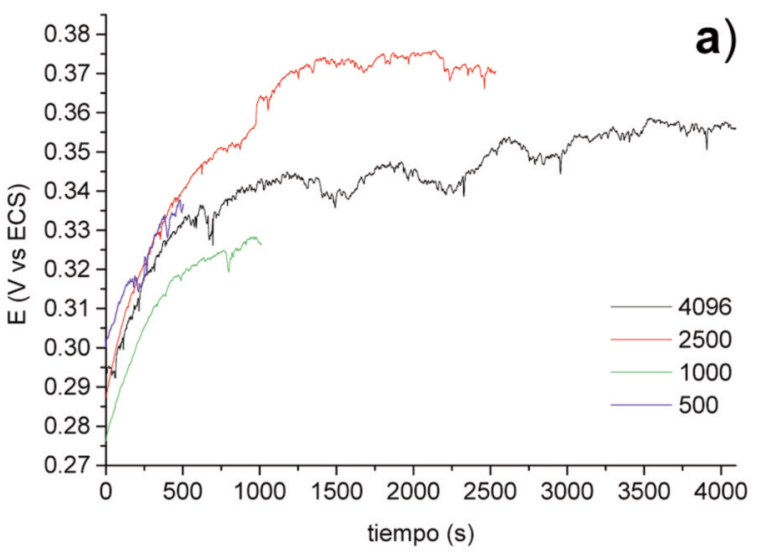

con intensidades bajas de $4 \times 10^{-6} \mathrm{~A} / \mathrm{cm}^{2}$; lo cual es característico de la nucleación de picaduras en medios corrosivos como el $\mathrm{FeCl}_{3}$; a pesar de que los aceros inoxidables presentan películas de óxido pasivas debido a los elementos aleantes de su composición química ${ }^{[23]}$.

La forma en la que se presenta la nucleación y el crecimiento de la picadura se puede observar en las micrografías, donde a un tiempo de exposición de $500 \mathrm{~s}$, se encontraron nucleaciones de $1 \mu \mathrm{m}$, у a medida que el tiempo de exposición de la muestra en el medio aumenta, el tamaño de la picadura se incrementa, de tal manera que se encontraron picaduras con un tamaño aproximado de $4 \mu \mathrm{m}$ aproximadamente para un tiempo de $4.096 \mathrm{~s}$. En el análisis de las muestras por MEB se observó que el número aproximado de picaduras correspondía a los transitorios observados en las series de tiempo. Algunas de estas crecieron y se propagaron, en tanto otras se repasivaron.

La figura 3 presenta las series de tiempo en potencial y corriente respectivamente, para las mediciones de ruido electroquímico con 500, 1.000, 2.500 y 4.096 datos muestreados, para el acero inoxidable austenítico 316 en la solución de $\mathrm{FeCl}_{3}$ al $6 \%$.

En la serie de tiempo en potencial figura 3 a), se observa que las fluctuaciones inician en potenciales nobles, para después disminuir hacia potenciales activos (contrario al caso anterior), causado posiblemente por la disolución de la película pasiva $^{[24]}$. Sin importar el número de datos muestreados, todos siguen una misma tendencia, sin embargo se aprecia que en la lectura de 4.096 datos, el acero inoxidable 316 comienza a estabilizar su potencial, lo cual indica que se está pasivando. En

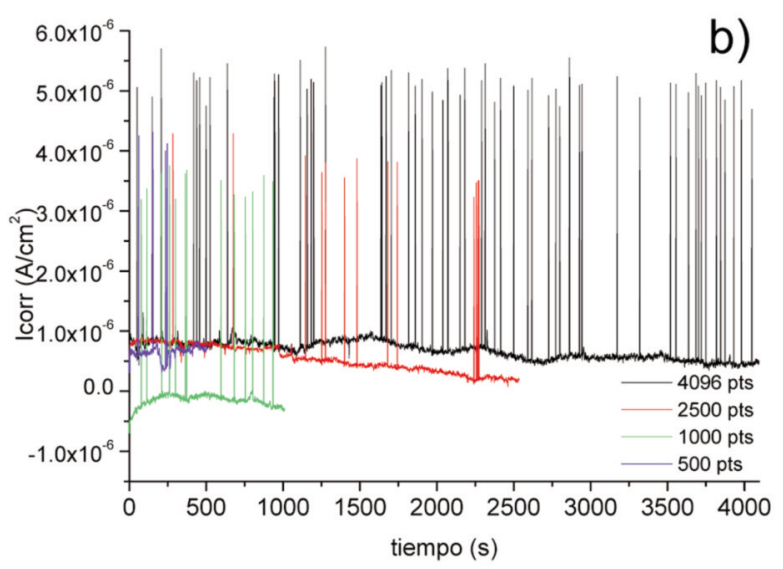

Figura 1. Series de tiempo en potencial (a) y corriente (b) para el acero dúplex 2205 en solución de $\mathrm{FeCl}_{3}$ al $6 \%$ en peso.

Figure 1. Potential (a) and current (b) time series for duplex 2205 steel in $6 \% \mathrm{w} \mathrm{FeCl}_{3}$ solution. 


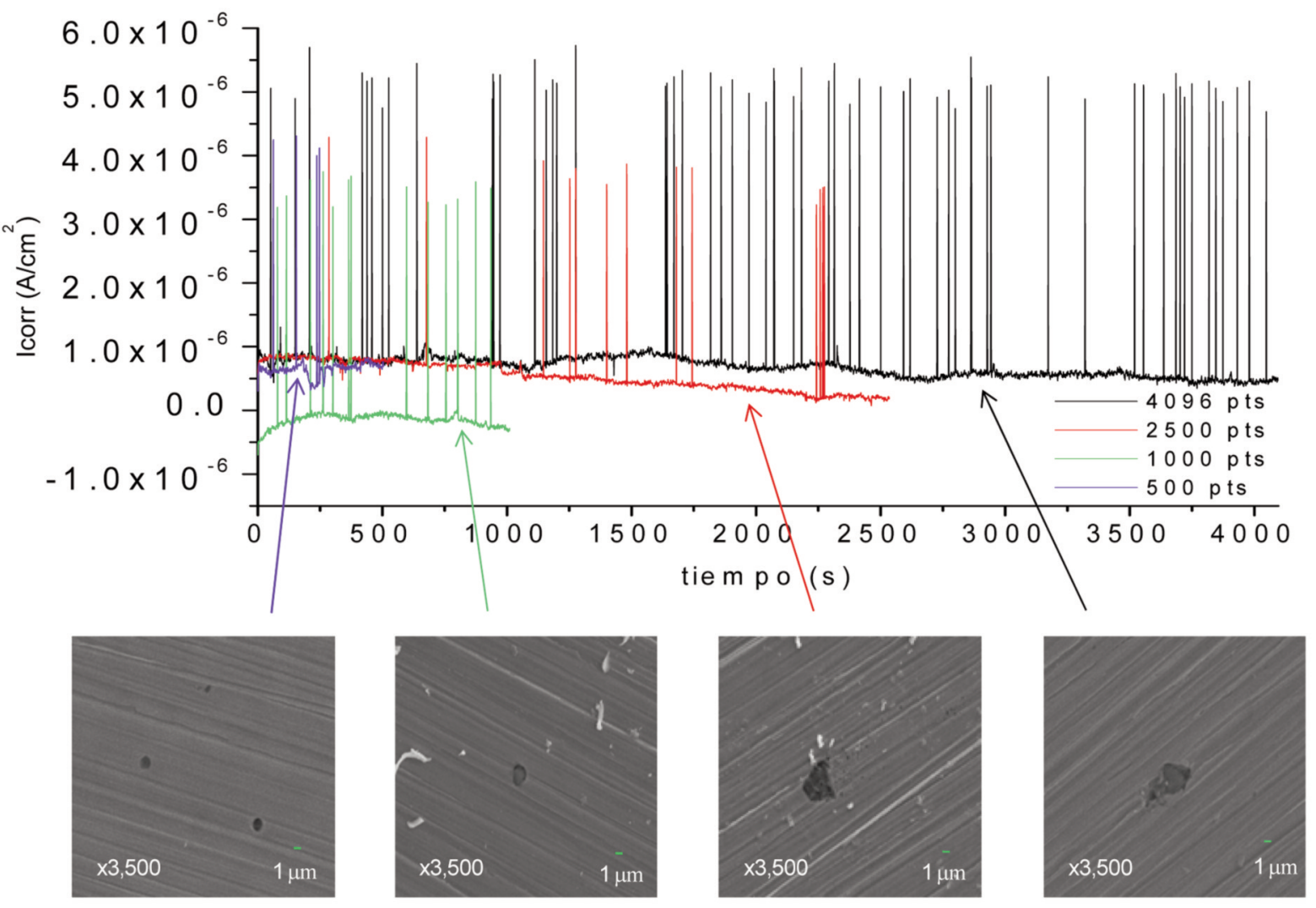

Figura 2. Serie de tiempo en corriente para el acero dúplex 2205 en solución de $\mathrm{FeCl}_{3}$ al $6 \%$; con $500,1.000,2.500$ y 4.096 datos, y sus correspondientes fotomicrografías tomadas por MËB a 3.500X.

Figure 2. Current time series for duplex 2205 steel in $6 \%$ w FeCl ${ }_{3}$ solution; with 500, 1000, 2500 y 4096 dates, and its corresponding SEM images (3500X).
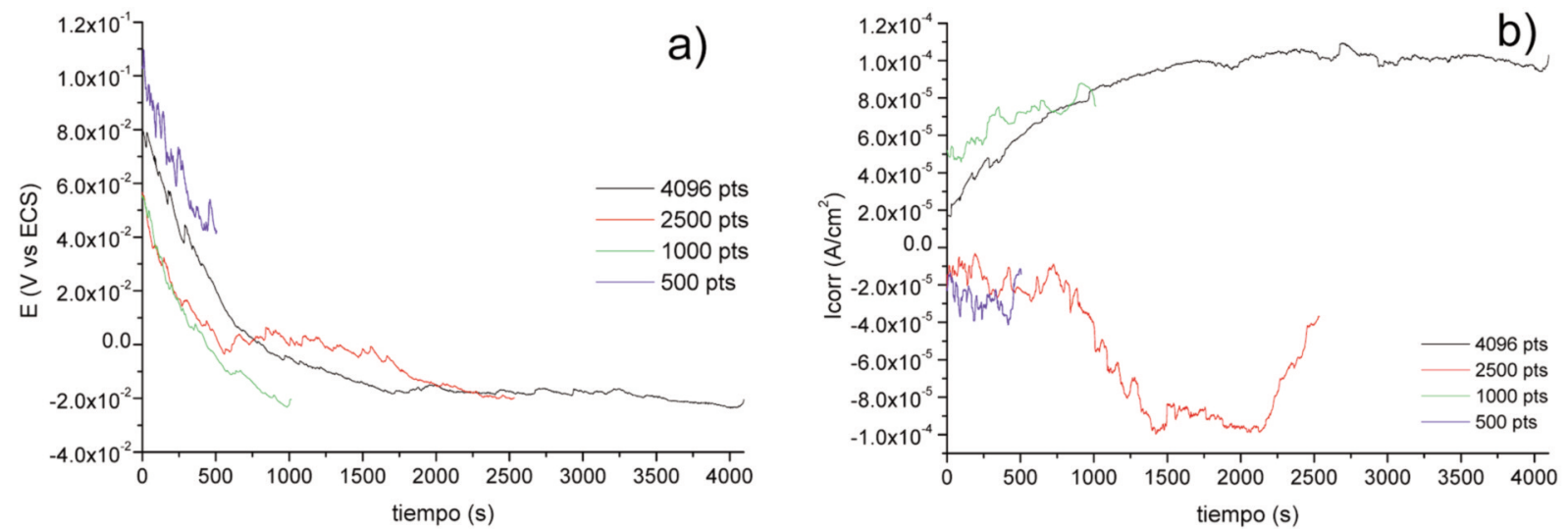

Figura 3. Series de tiempo en potencial (a) y corriente (b) para el acero inoxidable austenítico 316 en solución de $\mathrm{FeCl}_{3}$ al $6 \%$.

Figure 3. Potential (a) and current (b) time series for 316 austenitic stainless steel in $6 \%$ w de $\mathrm{FeCl}_{3}$ solution.

la serie de tiempo en corriente figura $3 \mathrm{~b}$ ), con 500 , 1.000 y 4.096 datos muestreados, se observa un incremento que corresponde a una disolución local de la película pasiva del acero inoxidable, con tendencia a la repasivación como se puede observar en la serie con 4.096 datos, donde el consumo de 


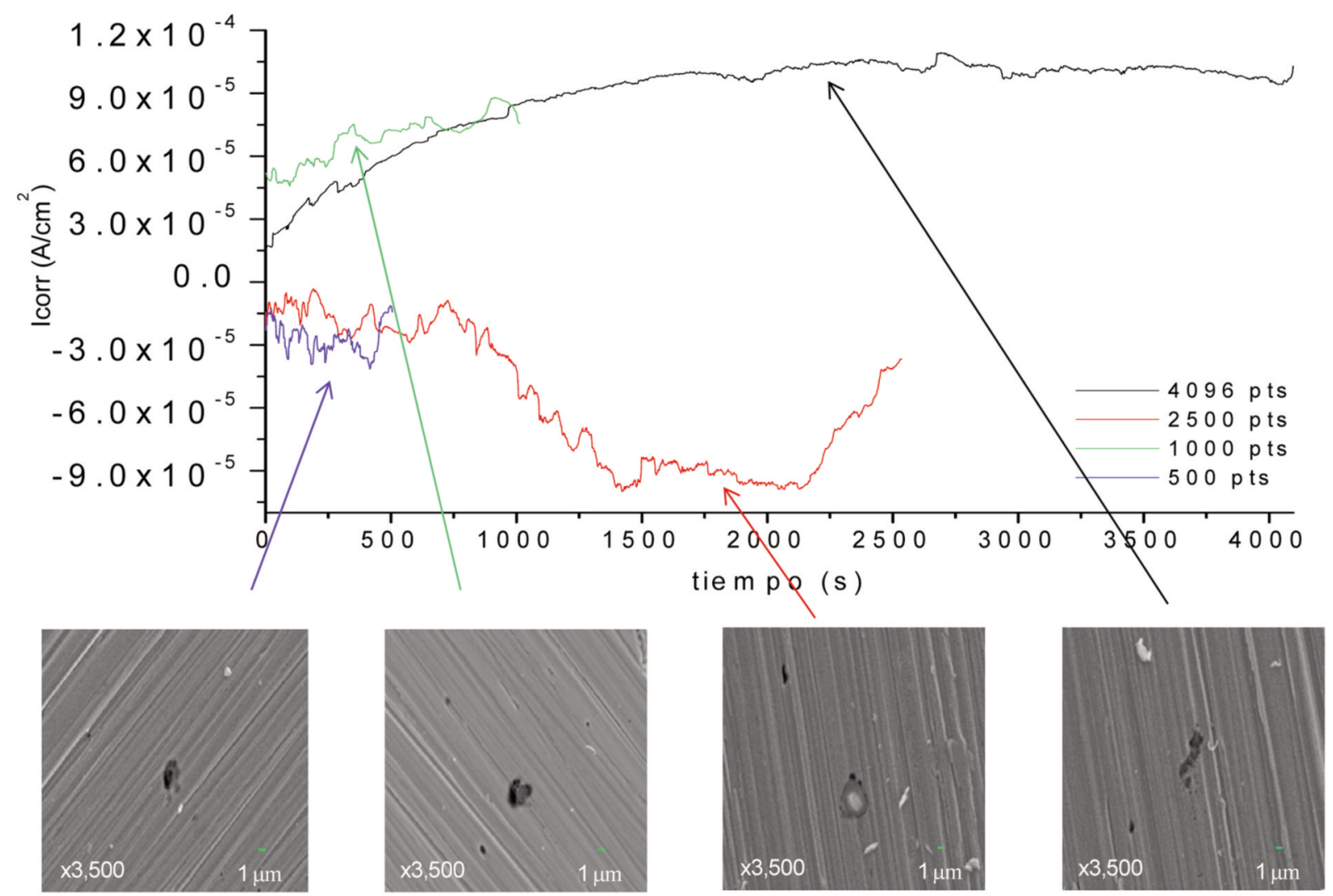

Figura 4. Serie de tiempo en corriente para el acero inoxidable austenítico 316 en solución de $\mathrm{FeCl}_{3}$ al $6 \%$ con 500,1.000, 2.500 y 4.096 datos, con sus correspondientes fotomicrografías tomadas por MEB a 3.500X.

Figure 4. Current time series for 316 austenitic stainless steel in $6 \% \mathrm{w}$ de $\mathrm{FeCl}_{3}$ solution with 500, 1000, 2500 y 4096 dates, and its corresponding SEM images (3500X).

corriente no sigue aumentando y tiende a estabilizarse, quizá al no aumentar el número de sitios $\operatorname{activos}^{[25]}$.

La figura 4, muestra la serie de tiempo en corriente del acero inoxidable 316 con sus respectivas fotomicrografías para los 500, 1.000, 2.500 y 4.096 datos muestreados. A diferencia del acero dúplex 2205 en la misma solución de $\mathrm{FeCl}_{3}$, en este material no se aprecian transitorios de amplitudes que se alejen significativamente de la media, sin embargo, sí se identifican incrementos en corriente seguidos de una disminución en la misma. En la fotomicrografía correspondiente a los 500 datos se observa una picadura, y alrededor de ella una serie de nucleaciones o picaduras metaestables, que por lo observado en las fotomicrografías se sabe que no todas se llegan a desarrollar. Se observó un crecimiento de las picaduras de hasta $6 \mu \mathrm{m}$ de largo y $3 \mu \mathrm{m}$ de ancho aproximadamente, presentando forma irregular que crece a lo largo del desbaste para un tiempo de experimentación de $4.096 \mathrm{~s}$.
En la serie de tiempo en potencial figura 5 a) y corriente figura $5 \mathrm{~b}$ ) sin tendencia para el acero inoxidable dúplex 2205 en la solución de $\mathrm{NaCl}$ al 3,5\%, se manifiesta actividad de corrosión localizada, específicamente una serie de nucleaciones de picaduras. En la figura 5 a) se observa una tendencia por parte del potencial hacia valores más nobles y un estado de pasivación; la tendencia se identifica claramente en la gráfica de 4.096 datos, aunque se puede identificar su comportamiento desde los $800 \mathrm{~s}$ aproximadamente. Se puede observar también que a un tiempo de entre 500 y 800 s, existe una caída en el potencial, mostrando correspondencia con las fluctuaciones observadas en la serie de tiempo de corriente, figura $5 \mathrm{~b}$ ), lo que indicaría el desarrollo de una picadura metaestable. Se debe notar que en la corriente a los $2.500 \mathrm{~s}$, aumenta la frecuencia de los transitorios súbitos de mayor amplitud.

Para el acero dúplex 2205 en solución de $\mathrm{NaCl}$, se presenta en la figura 6 , correspondiente a la serie de tiempo en corriente junto con las fotomicro- 

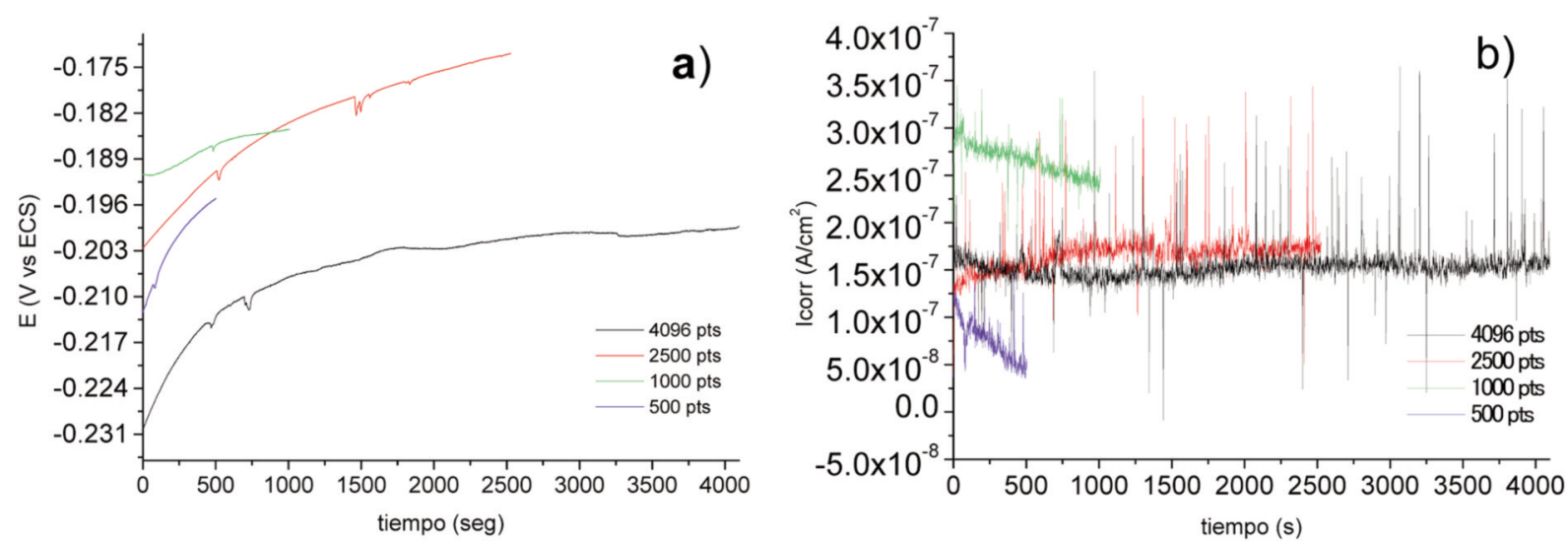

Figura 5. Series de tiempo en potencial (a) y corriente (b) para el acero dúplex 2205 en solución de $\mathrm{NaCl}$ al 3,5\%.

Figure 5. Potential (a) and current (b) time series for duplex 2205 steel in $3.5 \% \mathrm{NaCl}$ solution.

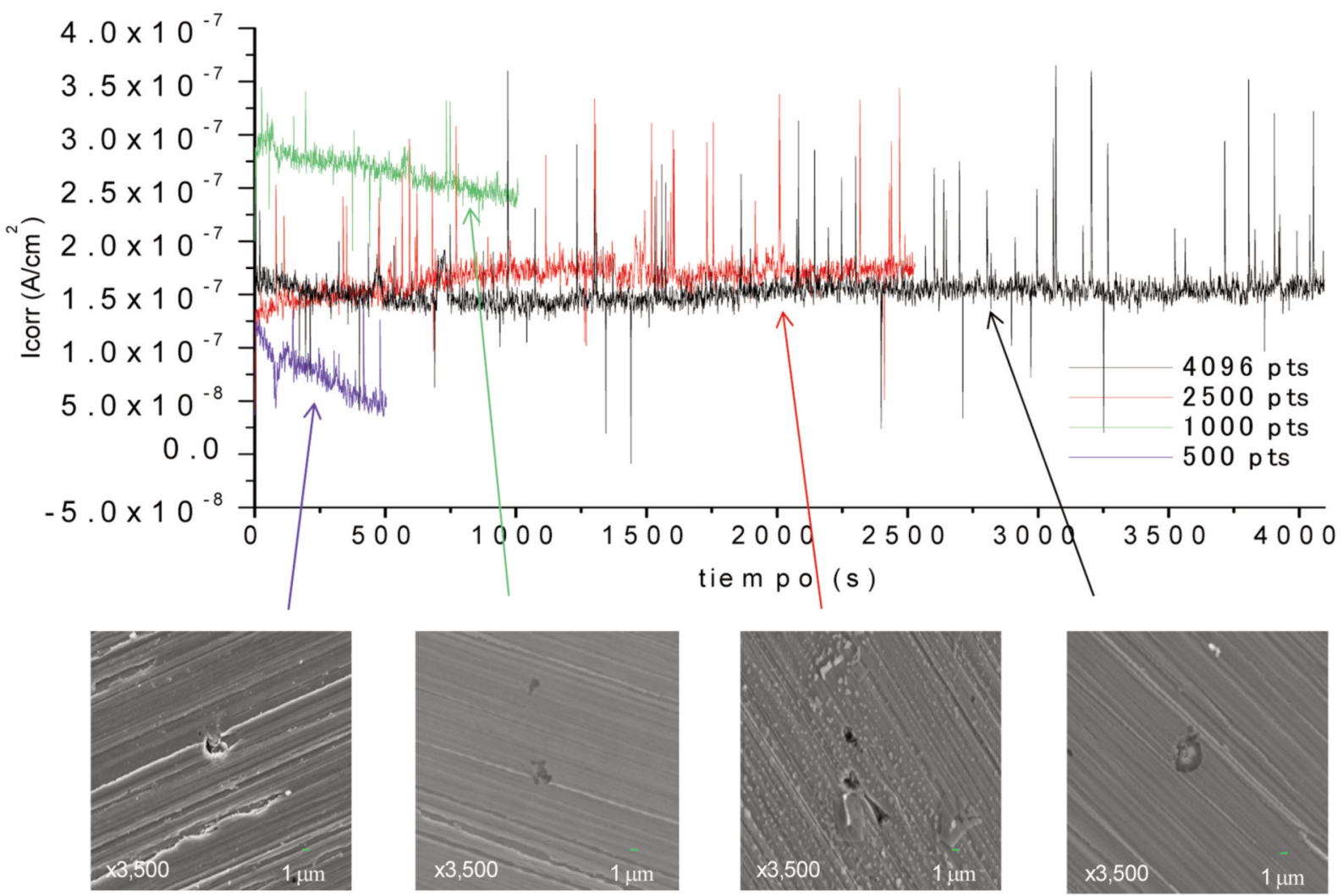

Figura 6. Serie de tiempo en corriente para el acero dúplex 2205 en solución de $\mathrm{NaCl}$ al 3,5 $\%$ con $500,1.000,2.500$ y 4.096 datos, y sus correspondientes fotomicrografías tomadas por MEB a 3.500X.

Figure 6. Current time series for duplex 2205 steel in $3.5 \%$ NaCl solution; with 500, 1000, 2500 y 4096 dates, and its corresponding SEM images (3500X).

grafías tomadas mediante MEB. La amplitud de los transitorios en las serie de tiempo con 500, 1.000, 2.500 y 4.096 puntos es la misma, identificándose claramente el ruido de fondo y distinguiéndose transitorios con una amplitud aproximada de $2,5 \times 10^{-7} \mathrm{~A} / \mathrm{cm}^{2}$; los transitorios presentes tienen 
un tiempo de vida muy corto y son en dirección tanto anódica como catódica, lo cual indica que existe una ruptura de la película pasiva en zonas localizadas, originado posiblemente por la concentración de iones cloruro.

En el análisis mediante MEB de la muestra correspondiente al monitoreo de 500 puntos, se observó muy poca nucleación de picaduras, y las que se detectaron, se encontraban de forma muy superficial en la muestra. En comparación con los resultados obtenidos en $\mathrm{el} \mathrm{FeCl}_{3}$ para este material, las intensidades en los transitorios de corriente son menores $\left(2,5 \times 10^{-7} \mathrm{~A} / \mathrm{cm}^{2}\right)$, lo cual se puede verificar en las fotomicrografías de la figura 6 , las cuales tienen menor tamaño y están de forma superficial para cualquiera de las series.

Los registros de potencial (a) y corriente (b) en el tiempo, para el acero inoxidable 316 en una solución de $\mathrm{NaCl}$ al 3,5 \%, se muestran en la figura 7. Se observa en la figura 7 a) un comportamiento con decremento de las fluctuaciones de potencial para cualquier número de datos muestreados. De la misma manera que en la solución de $\mathrm{FeCl}_{3}$ para el acero inoxidable 316, la tendencia es hacia potenciales activos. Se identifican varios decrementos en el transcurso del experimento a $4.096 \mathrm{~s}$, seguidos por una recuperación del potencial y este es un comportamiento correspondiente con las fluctuaciones presentes en la serie de tiempo de corriente, figura 7 b), en donde se observa un aumento insignificante en la corriente.

Analizando conjuntamente la serie de tiempo de corriente y las fotomicrografías de la figura 8, correspondientes al acero inoxidable 316 para los datos muestreados durante 500,1.000, 2.500 y $4.096 \mathrm{~s}$, se observa que existen transitorios que se repiten inde- pendientemente del número de datos elegidos, aunque no precisamente al mismo tiempo, lo cual da una repetitividad de las pruebas.

Los transitorios mostrados en la figura 8 exhiben la forma típica de nucleación de picaduras, un aumento rápido en la corriente seguido por un decremento hacia la media. La amplitud de los transitorios característicos de nucleación, es en promedio de $2,5 \times 10^{-7} \mathrm{~A} / \mathrm{cm}^{2}$, sin embargo existen algunos que alcanzan una amplitud de hasta $4 \times 10^{-7} \mathrm{~A} / \mathrm{cm}^{2}$. En comparación con lo observado en el $\mathrm{FeCl}_{3}$ en la serie de tiempo de corriente mediante el análisis de MEB, existe una menor densidad de nucleaciones de picado. Las nucleaciones de picaduras se encuentran de forma superficial en la muestra, y presentan una forma irregular de aproximadamente $6 \mu \mathrm{m}$ de largo y $2 \mu \mathrm{m}$ de ancho para el experimento con $4.096 \mathrm{~s}$.

Los resultados de los parámetros obtenidos (resistencia de ruido, índice de localización, densidad de corriente de corrosión y tipo de corrosión ${ }^{[26]}$, de los ensayos electroquímicos, se muestran en la tabla II, y confirman los obtenidos mediante el análisis por MEB. Para obtener los parámetros electroquímicos se recurrió al método estadístico de mínimos cuadrados, calculados para el experimento con 4096 datos. El cálculo de la resistencia de ruido (ec. 1) se realizó mediante la razón de la desviación estándar del ruido en potencial $(\sigma v)$ sobre la desviación estándar del ruido en corriente $(\sigma i)^{[27-29]}$ :

$$
\mathrm{R}_{\mathrm{n}}=\frac{\sigma \mathrm{V}}{\sigma \mathrm{i}}
$$

Los datos de $R n$ fueron usados para calcular la velocidad de corrosión (ec. 2), en analogía con la ecuación de Stern - Geary ${ }^{[30]}$ :
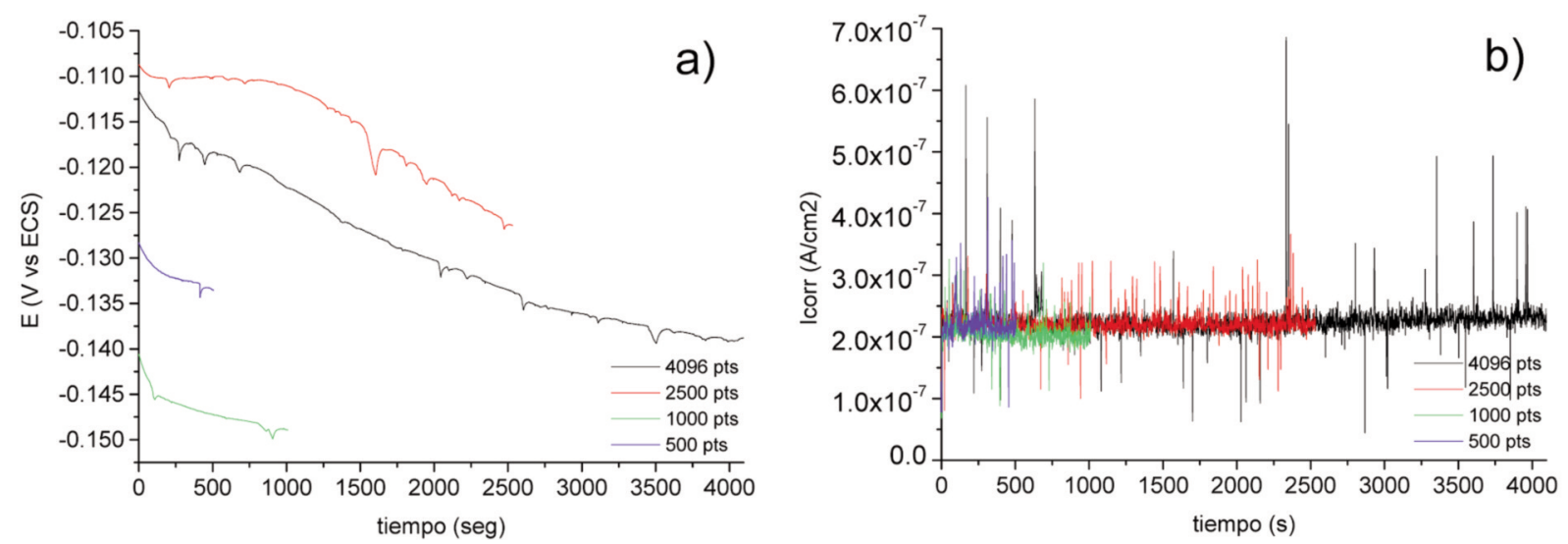

Figura 7. Series de tiempo en potencial (a) y corriente (b) del acero inoxidable austenítico 316 en solución de $\mathrm{NaCl} 3,5 \%$.

Figure 7. Potential (a) and current (b) time series for 316 austenitic stainless steel in $3.5 \% \mathrm{NaCl}$ solution. 


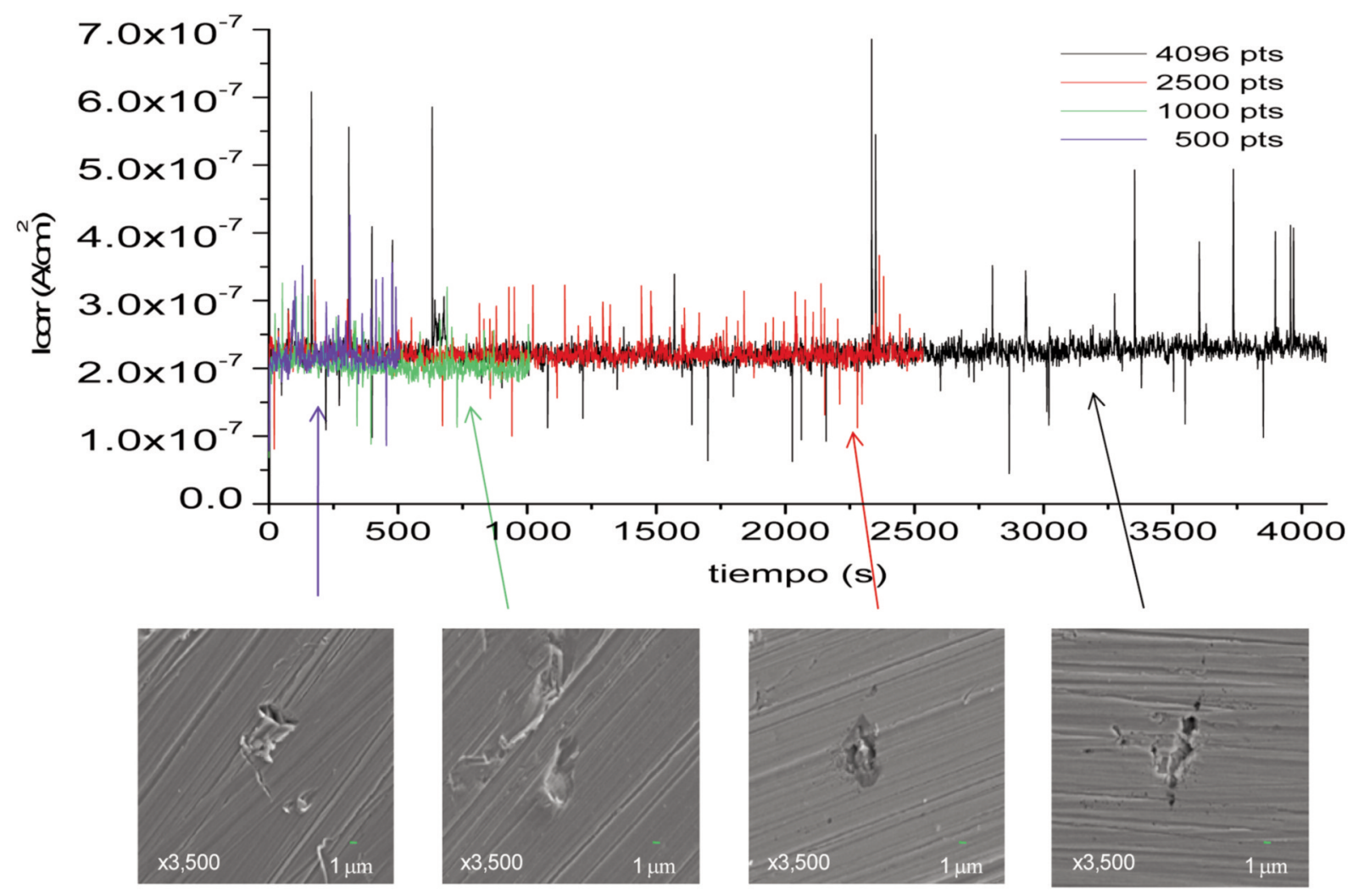

Figura 8. Serie de tiempo en corriente para el acero inoxidable austenítico 316 en solución de $\mathrm{NaCl}$ al 3,5 \% con 500,1.000, 2.500 y 4.096 datos, y sus correspondientes fotomicrografías tomadas por MEB a 3.500X.

Figure 8. Current time series for 316 austenitic stainless steel in $3.5 \% \mathrm{NaCl}$ solution with 500 , 1000, 2500 y 4096 dates, and its corresponding MEB images (3500X).

$$
\text { icorr }=\frac{\mathrm{B}}{\mathrm{Rn}}
$$

El índice de localización (ec. 3), el cual es un parámetro que evalúa la variación del ruido en corriente y la compara con el valor medio, se calculó a través de la razón de la desviación estándar en corriente $(\sigma i)$ y la raíz media cuadrática de la corriente (Irms) $)^{[15]}$.

$$
\mathrm{IL}=\frac{\sigma \mathrm{i}}{\mathrm{I}_{\mathrm{rms}}}
$$

Siendo el dúplex 2205 el que presenta el ataque más localizado de acuerdo con esto.

Tabla II. Resultados de las pruebas electroquímicas y tipo de corrosión[30]

\begin{tabular}{|c|c|c|c|c|c|}
\hline \multirow{2}{*}{ Material } & \multirow{2}{*}{ Solución } & \multicolumn{4}{|c|}{ Parámetros Electroquímicos } \\
\hline & & $\operatorname{Rn}\left(\Omega-\mathrm{cm}^{2}\right)$ & $I_{\text {corr }}\left(A / \mathrm{cm}^{2}\right)$ & IL & Tipo de Corrosión \\
\hline Duplex 2205 & $\begin{array}{l}\mathrm{FeCl}_{3} \\
\mathrm{NaCl}\end{array}$ & $\begin{array}{l}7.965,6 \\
54.768\end{array}$ & $\begin{array}{r}3,26 * 10^{-6} \\
4.7473 * 10^{-7}\end{array}$ & $\begin{array}{l}0,6017 \\
0,1118\end{array}$ & $\begin{array}{l}\text { Localizada } \\
\text { Localizada }\end{array}$ \\
\hline SS 316 & $\begin{array}{l}\mathrm{FeCl}_{3} \\
\mathrm{NaCl}\end{array}$ & $\begin{array}{r}1.582 \\
21.307\end{array}$ & $\begin{array}{r}1,64 * 10^{-5} \\
1.2202 * 10^{-6}\end{array}$ & $\begin{array}{l}0,2855 \\
0,1013\end{array}$ & $\begin{array}{l}\text { Localizada } \\
\text { Localizada }\end{array}$ \\
\hline
\end{tabular}

Table II. Electrochemical test results and proposed corrosion types ${ }^{[30]}$ 


\section{CONCLUSIONES}

- En todas las series de tiempo de potencial y corriente los transitorios presentan repetibilidad, y son característicos de nucleación de picaduras, o picaduras metaestables. Estos transitorios son independientes del número de datos muestreados y son confirmados por el análisis en MEB, el cual muestra correlación entre la densidad de nucleaciones de picadura y el número de transitorios en la serie de tiempo para cada experimento.

- La amplitud de los transitorios está relacionada con el tamaño de la nucleación de la picadura. Para el acero dúplex 2205 en la solución de $\mathrm{FeCl}_{3}$, se identificaron transitorios con un tamaño promedio de $4 \times 10^{-6} \mathrm{~A} / \mathrm{cm}^{2}$, relacionándose con una nucleación de $1 \mu \mathrm{m}$. En el inoxidable austenítico en la solución de $\mathrm{FeCl}_{3}$ se observaron amplitudes de $4,3 \times 10^{-6} \mathrm{~A} / \mathrm{cm}^{2}$ en promedio, con una correspondencia en el tamaño de la nucleación de 1,5 $\mu \mathrm{m}$. En las soluciones de $\mathrm{NaCl}$ para ambos aceros, se presentaron amplitudes de un orden de magnitud menor a las observadas en la solución de $\mathrm{FeCl}_{3}$, y las nucleaciones de picaduras se encontraron de forma superficial en la muestra, a diferencia de las encontradas en las soluciones de $\mathrm{FeCl}_{3}$, las cuales eran mayores. En el caso del acero dúplex 2205 en $\mathrm{NaCl}$ el promedio de las amplitudes es de $1,5 \times 10^{-7} \mathrm{~A} / \mathrm{cm}^{2}$, y corresponde a $1 \mu \mathrm{m}$ en el tamaño de la nucleación; en tanto que para el inoxidable austenítico 316 en dicha solución, se observaron también amplitudes de $1,5 \times 10^{-7} \mathrm{~A} / \mathrm{cm}^{2}$, pero con 1,5 $\mu \mathrm{m}$ de tamaño.El acero dúplex 2205 fue el que presentó mayor resistencia de ruido $\left(54768 \Omega-\mathrm{cm}^{2}\right)$ en el $\mathrm{NaCl}$, y el inoxidable 316 presentó la menor resistencia $\left(1.582 \Omega-\mathrm{cm}^{2}\right)$ en la solución de $\mathrm{FeCl}_{3}$.

- El índice de localización para los aceros inoxidables 316 y dúplex 2205 en las dos soluciones $\left(\mathrm{FeCl}_{3}\right.$ y $\left.\mathrm{NaCl}\right)$, corresponde a un tipo de corrosión localizada, presentando el mayor índice el dúplex 2205 en la solución de $\mathrm{FeCl}_{3}$, y el menor índice el acero inoxidable 316 en la solución de $\mathrm{NaCl}$.

\section{Agradecimientos}

Los autores agradecen a CONACYT por el apoyo económico para llevar a cabo los estudios de doctorado, con el número de beca 160250 .

\section{REFERENCIAS}

[1] P. Marcus e I. Olefjord, Corros. Sci. 28(1988) 589.

[2] Omar A. Hazzazi, Ayman M. Zaky, Mohammed A. Amin y Sayed S. Abd El Rehim, Int. J . Electrochem. Sci. 3 (2008) 489-508.

[3] Z. Szklarska-Smialowska., Pitting Corrosion of Metals, National Association of Corrosion Engineers, Houston, Texas, EE.UU, 1986, p. 1.

[4] K. Darowicki, A. Mirakowski, S. Krakowiak, Corros. Sci. 45 (2003) 1.747-1756.

[5] A. Pardoa, M.C. Merinoa, A.E. Coyb, F. Viejob, R. Arrabalb y E. Matykinab, Corros. Sci. 6 (2008) 1.796-1800.

[6] E. Sarmiento, J. G. González-Rodriguez, J. Uruchurtu, O.Sarmiento, M. Menchaca, Int. J. Electrochem. Sci. 4 (2009) 144-155.

[7] M. G. Pujar, T. Anita, H. Shaikh, R. K. Dayal y H. S. Khatak, Int. J. Electrochem. Sci. 2 (2007) 301-310.

[8] U. Bertocci y F. Huet, Corrosion 51 (1995) 131.

[9] U. Bertocci, C. Gabrielli. F. Huet, y M. Keddam, J. Electrochem. Soc. 144, (1997) 31.

[10] P. R. Roberge, Corrosion, 50 (1994) 502.

[11] G. Gusmano, G. Montespereli, S. Pacetti, A. Petitti, y A. D'Amico, Corrosion 53 (1997) 860.

[12] J. W. Isaac y K. R. Hebert, J. Electrochem. Soc. 146 (1999) 502.

[13] C. Monticelli, G. Brunoro, A. Frignani, y G. Trabanelli, J. Electrochem. Soc. 139 (1992) 706.

[14] R. A. Cottis, M. A. Al-Ansari, G. Bagley, y A. Pettiti, Mater. Sci. Forum 289-292 (1998) 741.

[15] K. Hladki and J.L. Dawson, Corros. Sci. 3 (1982) 231-237.

[16] S. Girija, U. Kamachi Mudali, V.R. Raju, R.K. Dayal, H.S. Khatak y Baldeb Raj., Mat. Sci. Eng. 407 (2005) 188.

[17] J. Smulko, K. Darowicki y A. Zielinski, Elect. Comm. 4 (2002) 388.

[18] Helmuth Sarmiento Klapper y Joachim Goellner, Corros. Sci. 51 (2009) 144-150.

[19] ASTM International, G 199-09, Standard Guide for Electrochemical Noise Measurement, ASTM International 2009, pp. 14-20.

[20] F.M. Almeraya-Calderon. Ph. D. Thesis. Centro de Investigación en Materiales Avanzados. S. C., Chihuahua, Chi, México. 1998.

[21] U. Bertocci, J electrochem.l Soc. 128 (1981) 520.

[22] Helmuth Sarmiento Klapper, Joachim Goellner y Andreas Heyn, Corros.Sci. 52 (2010) 1.362-1.372.

[23] L. Speckert y G.T. Burstein, Corros. Sci. 53 (2011) 534-539.

[24] J. A. Wharton, R. J. K. Wood y B. G. Mellor, Corros. Sci. 45 (2003) 97-122. 
[25] U. Bertocci y J. Kruger, Surf. Sci. 101 (1980) 608.

[26] Botana Pedemonte, M. Marcos Bárcena y A. Aballe Villero, Ruido Electroquímico, Métodos de Análisis, Septem Ediciones, Oviedo, España, 2002, p. 25.

[27] F. Barragán, R. Guardián, C. Menchaca, I. Rosales y J. Uruchurtu, Int. J. Electrochem. Sci. 5 (2010) $1.799-1.809$.

[28] J. R. Kearns, J. R. Scully, P. R. Roberge, D.L. Reichert y J. L. Dawson, Electrochemical Noise
Measurement, for Corrosion Applications, STP1277, ASTM 1996, pp. 206-208.

[29] R. Urzua, J. Siqueiros, L. Morales, I. Rosales y J. Uruchurtu, Portugaliae Electrochim. Acta, 27 (2009) 127.

[30] M. Stern., A. Geary, Journal Electrochem. Soc. 104 (1957) 56.

[31] Kelly, Inman y Hudson., Electrochemical Noise Measurement for Corrosion Applications, ASTM SP-1277. ASTM 1996, pp. 34-49. 